Review Article

\title{
Evaluation of Serum Des-Gamma-Carboxy Prothrombin for the Diagnosis of Hepatitis B Virus-Related Hepatocellular Carcinoma: A Meta-Analysis
}

\author{
Jiu Chen, Guolin Wu, and Youdi Li (iD \\ Department of Traditional Chinese Medicine, The First Affiliated Hospital, College of Medicine, Zhejiang University, \\ \#79 Qingchun Road, Hangzhou, Zhejiang Province, China 310003
}

Correspondence should be addressed to Youdi Li; linyunju@zju.edu.cn

Received 25 May 2018; Revised 2 August 2018; Accepted 29 August 2018; Published 4 October 2018

Academic Editor: Ralf Lichtinghagen

Copyright ( 2018 Jiu Chen et al. This is an open access article distributed under the Creative Commons Attribution License, which permits unrestricted use, distribution, and reproduction in any medium, provided the original work is properly cited.

\begin{abstract}
Aim. To explore the diagnostic efficacy of des-gamma-carboxy prothrombin (DCP) in hepatitis B virus- (HBV-) related hepatocellular carcinoma (HCC). Methods. A retrospective study of 459 cases from June 2016 to March 2018 was undertaken, and records of the DCP levels were extracted. The sensitivity, specificity, and cutoff points were calculated using SPSS 17.0 software. A systematic search in PubMed, Web of Science, and the Cochrane Central Register of Controlled Trials was performed for articles published in English from 1997 to 2017, focusing on serum DCP for HBV-related HCC. Data on sensitivity, specificity, the positive likelihood ratio (PLR), negative likelihood ratio (NLR), and diagnostic odds ratio (DOR) were extracted from five studies by systematic search and one study of our own. The summary receiver operating characteristic (sROC) curve was obtained, and the area under the receiver operating characteristic (AUROC) curve was calculated. Results. The pooled sensitivity, specificity, PLR, NLR, and DOR were 0.71 (95\% CI: 0.59, 0.80), 0.93 (95\% CI: 0.87, 0.96), 9.5 (95\% CI: 5.2, 17.5), 0.32 (95\% CI: 0.22, 0.46), and 30 (95\% CI: 13, 72), respectively. The AUROC curve was 0.91 (95\% CI: $0.88,0.93$ ). Conclusions. In the diagnosis of HBV-related hepatocellular carcinoma (HCC), DCP is an ideal marker that should be considered for surveillance purposes.
\end{abstract}

\section{Introduction}

Primary liver cancer is the fifth most common cancer and is responsible for the second most common cancer-related deaths worldwide [1]. Hepatocellular carcinoma (HCC) alone accounts for approximately $80 \%$ of all cases [2] and is one type of malignancy with a very poor prognosis [1]. Early identification can improve the prognosis. However, HCC usually arises against the background of liver damage, and the tumors are usually relatively large before any symptoms become evident [3]. Previous studies have shown that if patients are diagnosed at an early stage, the 5-year survival rate can be above $70 \%$ [4]. If patients are diagnosed at a late stage, however, the 5-year survival rate is less than 5\% [5]. These features make early identification both difficult and important.
Ultrasonography (US) alone, without concurrent detection of serum alpha fetoprotein levels, has been recommended for the surveillance of HCC, according to the representative guidelines of the American Association for the Study of Liver Diseases in 2010 [6] and the European Association for the Study of the Liver in 2012 [7]. However, interpretation of the sonogram is dependent on the expertise of the operator and quality of the equipment [8] and can be disrupted by other conditions of the liver, such as cirrhosis [9]. The overall sensitivity of US in this context is only $0.593[10]$.

The identification of new markers for the diagnosis of HCC is urgently required. Serum des-gamma-carboxy prothrombin (DCP), also known as prothrombin-induced by vitamin $\mathrm{K}$ absence-II (PIVKA-II), was first reported by Liebman et al. in 1984 [11]. Its value has been confirmed in 
the diagnosis of HCC in a series of clinical trials [12-20]. However, the sensitivity, specificity, and cutoff points in previous studies have been inconsistent, and in some instances, even conflicting [21]. One of the reasons for this disparity could be the differences in etiology and the fact that diagnostic values were mainly derived from various patients without homogeneous etiologies.

Hepatitis B virus (HBV) infection is the main causative factor of HCC, and more than half of all cases could be attributed to HBV infection worldwide [22]. Even with improvements in technologies, the morbidity and mortality of HBV-related HCC have still shown a steady increase [23].

Within the context of increased sensitivity, DCP has been considered for the diagnosis, treatment response, recurrence monitoring, and prognosis of HBV-related HCC [24-28]. However, the precise diagnostic efficacy has never been fully evaluated. We conducted this meta-analysis to elucidate this pertinent issue.

\section{Methods}

2.1. Retrospective Study. A total of 459 participants were retrospectively enrolled at the First Affiliated Hospital, College of Medicine, Zhejiang University, from June 2016 to March 2018. The study was approved by the Ethics Committees of the First Affiliated Hospital, College of Medicine, Zhejiang University.

\subsubsection{Criteria of Selection}

(1) Patients were hepatitis B surface antigen-positive $(>0.05 \mathrm{IU} / \mathrm{mL})$ and/or HBV DNA-positive (>30 IU/ $\mathrm{mL})$

(2) Serum DCP and indexes of HBV were both measured at the same time

(3) The DCP was measured by enzyme immunoassay

(4) The diagnosis of HCC was based on histological examination

\subsubsection{Criteria of Exclusion}

(1) The diagnosis of HCC was based on imaging characteristics

(2) Patients were also affected by other types of hepatitis virus infections, nonalcoholic steatohepatitis, alcoholic steatohepatitis, drug-induced hepatitis, or autoimmune hepatitis

(3) Patients had undergone liver transplantation

(4) Patients were taking vitamin $\mathrm{K}$ or warfarin within one week of DCP measurement

(5) Patients showed other evidence of other kinds of malignancies besides HCC

(6) Nodules in the liver could not have been confirmed; for example, the patients refused biopsy or surgery
(7) Patients were treated before DCP measurement

2.2. Literature Screening for Meta-Analysis. Considering that the diagnostic technique was revised in 1997, resulting in a much higher sensitivity [29], a systematic search was conducted from January 1, 1997, to December 31, 2017, by two investigators independently (Jiu Chen and Youdi Li). Searches for relevant studies were mainly conducted in PubMed, Web of Science, and the Cochrane Central Register of Controlled Trials.

The literature search strategy is summarized and depicted in Table 1. A flow diagram of the study selection process is summarized in Figure 1.

2.2.1. Levels of Literature Screening. Literature screening was performed at four levels, as follows.

(1) Level 1. Level 1 was derived from the three databases.

(i) PubMed

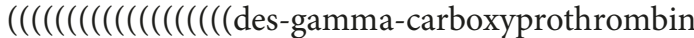
[Title/Abstract] OR ((des-gamma-carboxy [All Fields] AND "prothrombin"[MeSH Terms]) OR des-gamma-carboxy prothrombin [Title/Abstract])) OR ((des[All Fields] AND gamma-carboxy[All Fields] AND “prothrombin"[MeSH Terms]) OR des[All Fields] AND gamma-carboxy[All Fields] AND prothrombin [Title/Abstract])) OR Isoprothrombin [Title/Abstract]) OR prothrombin precursor [Title/Abstract]) OR PIVKA [Title/Abstract]) OR ((“"proteins”[MeSH Terms] OR “proteins"[All Fields] OR "protein"[All Fields]) AND induced [All Fields] AND ("vitamin k"[MeSH Terms] OR “vitamin k"[All Fields]) AND absence [All Fields]) OR (protein induced by vitamin $\mathrm{K}$ absence [Title/ Abstract] OR antagonists [Title/Abstract]))) OR ((descarboxylated [All Fields] AND "prothrombin"[MeSH Terms]) OR descarboxylated prothrombin [Title/Abstract])) OR ((acarboxy [All Fields] AND "prothrombin" [MeSH Terms]) OR acarboxy prothrombin [Title/Abstract])) OR (((“proteins”[MeSH Terms] OR “proteins"[All Fields] OR “protein”[All Fields]) AND induced [All Fields] AND ("vitamin k"[MeSH Terms] OR "vitamin k"[All Fields]) AND absence [All Fields]) OR (protein induced by vitamin $\mathrm{K}$ absence [Title/Abstract] OR antagonist-II [Title/ Abstract]))) OR PIVKA-II [Title/Abstract]) OR PIVKA II [Title/Abstract]) OR ((non-carboxylated [All Fields] AND “prothrombin"[MeSH Terms]) OR (non-carboxylated[All Fields] AND factor II[Title/Abstract]))) OR descarboxyprothrombin[Title/ Abstract]) OR des-gamma-carboxyprothrombin [Title/Abstract]) OR ((des-gamma-carboxy [All Fields] AND "prothrombin"[MeSH Terms]) OR des-gamma-carboxy prothrombin [Title/Abstract])) OR ((des [All Fields] AND gamma-carboxy [All Fields] AND "prothrombin"[MeSH Terms]) OR des [All Fields] AND gamma-carboxy [All Fields] AND prothrombin [Title/Abstract])) OR 


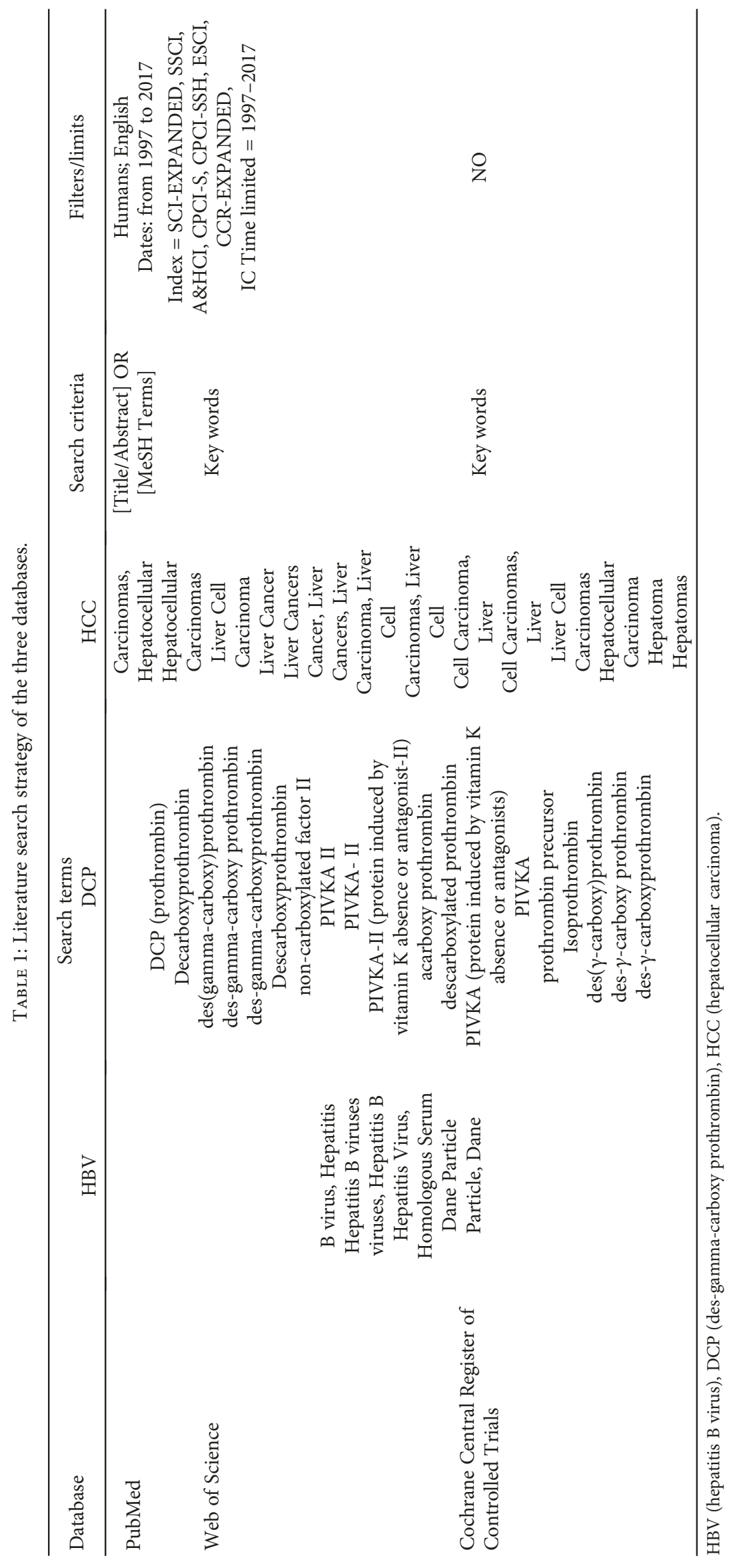




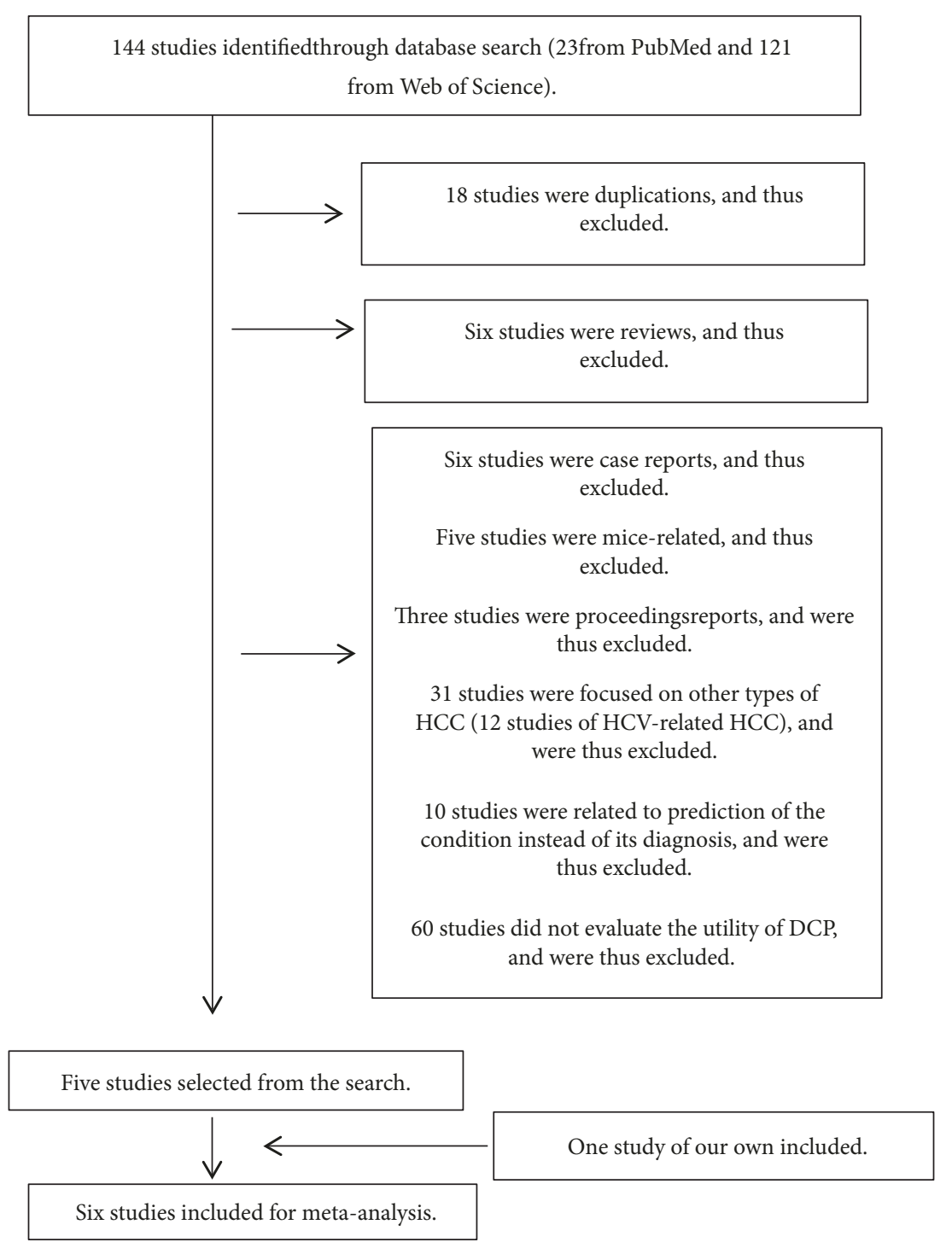

Figure 1: Literature screening was performed at four levels. Flowchart of literature research and study selection.

decarboxyprothrombin [Title/Abstract]) OR DCP [Title/Abstract]) AND ((()(“"hepatitis b virus"[MeSH Terms] OR (Particle [All Fields] AND Dane [Title/ Abstract])) OR ("hepatitis b virus"[MeSH Terms] OR Dane Particle[Title/Abstract])) OR ("hepatitis b virus" [MeSH Terms] OR (("hepatitis viruses" [MeSH Terms] OR ("hepatitis"[All Fields] AND “viruses"[All Fields]) OR "hepatitis viruses"[All Fields] OR ("hepatitis"[All Fields] AND "virus"[All Fields]) OR "hepatitis virus"[All Fields]) AND Homologous Serum[Title/ Abstract]))) OR ("hepatitis b virus"[MeSH Terms] OR (("virology"[Subheading] OR "virology"[All Fields] OR "viruses”[All Fields] OR “viruses"[MeSH Terms]) AND Hepatitis B[Title/Abstract]))) OR ("hepatitis b virus"[MeSH Terms] OR Hepatitis $\mathrm{B}$ viruses[Title/Abstract])) OR ("hepatitis b virus" [MeSH Terms] OR B virus, Hepatitis[Title/Abstract $])))$ AND $\quad((((()(((()((($ “carcinoma, hepatocellular”[MeSH Terms] OR Hepatomas[Title/
Abstract]) OR (“carcinoma, hepatocellular" [MeSH Terms] OR Hepatoma[Title/Abstract])) OR ("carcinoma, hepatocellular”[MeSH Terms] OR Hepatocellular Carcinoma[Title/Abstract])) OR ("carcinoma, hepatocellular”[MeSH Terms] OR Liver Cell Carcinomas[Title/Abstract])) OR ("carcinoma, hepatocellular"[MeSH Terms] OR ((“cells”[MeSH Terms] OR “cells"[All Fields] OR "cell”[All Fields]) AND ("carcinoma" [MeSH Terms] OR "carcinoma"[All Fields] OR “carcinomas"[All Fields]) AND Liver[Title/ Abstract]))) OR (“carcinoma, hepatocellular" [MeSH Terms] OR ((“cells"[MeSH Terms] OR “cells"[All Fields] OR “cell”[All Fields]) AND Carcinoma, Liver[Title/Abstract]))) OR ("carcinoma, hepatocellular" $[\mathrm{MeSH}$ Terms] OR (("carcinoma" $[\mathrm{MeSH}$ Terms] OR “carcinoma”[All Fields] OR “carcinomas”[All Fields]) AND Liver Cell[Title/Abstract]))) OR ("carcinoma, hepatocellular" [MeSH Terms] OR Carcinoma, Liver Cell[Title/Abstract])) OR ("liver 
neoplasms"[MeSH Terms] OR ((“neoplasms”[MeSH Terms] OR "neoplasms"[All Fields] OR "cancers"[All Fields]) AND Liver[Title/Abstract]))) OR ("liver neoplasms"[MeSH Terms] OR Cancer, Liver[Title/ Abstract])) OR ("liver neoplasms"[MeSH Terms] OR Liver Cancers[Title/Abstract])) OR ("liver neoplasms"[MeSH Terms] OR Liver Cancer[Title/ Abstract])) OR ("carcinoma, hepatocellular" [MeSH Terms] OR Liver Cell Carcinoma[Title/Abstract])) OR ("carcinoma, hepatocellular" [MeSH Terms] OR Hepatocellular Carcinomas[Title/Abstract])) OR ("carcinoma, hepatocellular"[MeSH Terms] OR (("carcinoma” [MeSH Terms] OR "carcinoma"[All Fields] OR "carcinomas"[All Fields]) AND Hepatocellular[Title/Abstract]))) AND ((“1997/01/01”[PDAT]: “2017/12/31”[PDAT]) AND "humans"[MeSH Terms] AND English[lang])

Altogether, 23 articles were included

\section{(ii) Web of Science}

Filters: Index=SCI-EXPANDED, SSCI, A\&HCI, CPCI-S, CPCI-SSH, ESCI, CCR-EXPANDED, IC Time limited $=1997-2017$

(1) Key words: (Carcinomas, Hepatocellular) OR Key words: (Hepatocellular Carcinomas) OR Key words: (Liver Cell Carcinoma) OR Key words: (Liver Cancer) OR Key words: (Liver Cancers) OR Key words: (Cancer, Liver) OR Key words: (Cancers, Liver) OR Key words: (Carcinoma, Liver Cell) OR Key words: (Carcinomas, Liver Cell) OR Key words: (Cell Carcinoma, Liver) OR Key words: (Cell Carcinomas, Liver) OR Key words: (Liver Cell Carcinomas) OR Key words: (Hepatocellular Carcinoma) OR Key words: (Hepatoma) OR Key words: (Hepatomas)

(2) Key words: (B virus, Hepatitis) OR Key words: (Hepatitis B viruses) OR Key words: (viruses, Hepatitis B) OR Key words: (Hepatitis Virus, Homologous Serum) OR Key words: (Dane Particle) OR Key words: (Particle, Dane)

(3) Key words: (DCP) OR Key words: (decarboxyprothrombin) OR Key words: (des(gamma-carboxy)prothrombin) OR Key words: (desgamma-carboxy prothrombin) OR Key words: (des-gamma-carboxyprothrombin) OR Key words: (descarboxyprothrombin) OR Key words: (non-carboxylated factor II) OR Key words: (PIVKA II) OR Key words: (PIVKA-II) OR Key words: (protein induced by vitamin $\mathrm{K}$ absence or antagonist-II) OR Key words: (acarboxy prothrombin) OR Key words: (descarboxylated prothrombin) OR Key words: (protein induced by vitamin $\mathrm{K}$ absence or antagonists) OR Key words: (PIVKA) OR Key words: (prothrombin precursor) OR Key words: (Isoprothrombin)
OR Key words: (des $(\gamma$-carboxy)prothrombin) OR Key words: (des- $\gamma$-carboxy prothrombin) OR Key words: (des- $\gamma$-carboxyprothrombin)
(4) (3) AND
(2) AND
(1): limited in the English language

Altogether, 137 studies were included. Two studies were excluded because they were not written in English, one was excluded because of duplication, four more were excluded because they reported on the proceedings of various conferences, and nine were excluded because they were reviews. Thus, 121 articles were included after the first level screening.

(iii) Cochrane Central Register of Controlled Trials

(1) (Carcinomas, Hepatocellular) or (Hepatocellular Carcinomas) or (Liver Cell Carcinoma) or (Liver Cancer) or (Liver Cancers) or (Cancer, Liver) or (Cancers, Liver) or (Carcinoma, Liver Cell) or (Carcinomas, Liver Cell) or (Cell Carcinoma, Liver) or (Cell Carcinomas, Liver) or (Liver Cell Carcinomas) or (Hepatocellular Carcinoma) or (Hepatoma) or (Hepatomas)

(2) (B virus, Hepatitis) or (Hepatitis B viruses) or (viruses, Hepatitis B) or (Hepatitis Virus, Homologous Serum) or (Dane Particle) or (Particle, Dane)

(3) (DCP) or (descarboxyprothrombin) or (des gamma-carboxy prothrombin) or (des-gammacarboxy prothrombin) or (des-gamma-carboxyprothrombin) or (decarboxyprothrombin) or (non-carboxylated factor II) or (PIVKA II) or (PIVKA-II) or (protein induced by vitamin $\mathrm{K}$ absence or antagonist-II) or (acarboxyl prothrombin) or (decarboxylated prothrombin) or (protein induced by vitamin $\mathrm{K}$ absence or antagonists) or (PIVKA) or (prothrombin precursor) or (Isoprothrombin) or (des $\gamma$-carboxy prothrombin) or (des- $\gamma$-carboxy prothrombin) or (des- $\gamma$-carboxyprothrombin)

(4) (1) and (2) and (3)

Altogether, 50 studies were included. 42 studies were reviews, and eight studies were unrelated, after screening of the title and abstract. Thus, no articles were included after the first-level screening.

(2) Level 2. Altogether, 144 studies were included and 18 duplications were excluded.

(3) Level 3. Altogether, 126 studies were included and six studies were excluded because they comprised reviews.

(4) Level 4. Five studies were eventually included from the searches conducted. Six studies were excluded because they were case reports; five studies were excluded because they were mice-related; three studies were excluded because they 
TABLE 2: Main characteristics of the studies included in the meta-analysis.

\begin{tabular}{lcccccccc}
\hline $\mathrm{N}$ & Author & Year & Country & Number of patients & Sensitivity & Specificity & Cutoff point (mAU/mL) & AUROC \\
\hline 1 & Young Joon Yoon et al. & 2009 & Korea & 206 & 0.519 & 0.97 & 40 & 0.777 \\
2 & Seung In Seo et al. & 2015 & Korea & 1255 & 0.739 & 0.897 & 0.854 \\
3 & Xiumei Wang et al. & 2017 & China & 113 & 0.5221 & 0.8149 & 30 & 0.756 \\
4 & Shujing Huang et al. & 2017 & China & 163 & 0.85 & 0.90 & 40 & 0.893 \\
5 & Xiao-Qiong Tang et al. & 2017 & China & 366 & 0.824 & 0.959 & 0.923 \\
6 & Chen et al. & 2018 & China & 459 & 0.720 & 0.937 & 40.5 & 0.863 \\
\hline
\end{tabular}

reported the proceedings of various conferences; 31 studies were excluded because they reported on other types of HCC (12 studies of hepatitis C virus- (HCV-) related HCC); 10 studies were excluded because they were related to the prediction of the condition, instead of its diagnosis; and 60 studies were excluded because they did not evaluate the utility of DCP.

2.2.2. Criteria of Selection for Meta-Analysis. Studies were included if they met the following inclusion criteria:

(1) Clinical studies that evaluated the efficiency of DCP for the diagnosis of $\mathrm{HBV}$-related $\mathrm{HCC}$, with a prospective or retrospective design. The efficiency of DCP was assessed either alone or in comparison with other tests

(2) The diagnosis of HCC was based on histological examination or the interpretation of appropriate imaging characteristics, as defined by accepted guidelines

(3) Selected studies detected the DCP concentration in serum

(4) Studies reported both the sensitivity and the specificity of DCP

\subsubsection{Criteria of Exclusion for Meta-Analysis}

(1) Diagnostic criteria and study population were ambiguous

(2) Studies detected DCP concentration in tissue and other body fluids

(3) Studies evaluated the efficiency of DCP for the prognosis of HCC

(4) Studies presented insufficient information to make a judgment

(5) Studies were published as reviews, letters, case reports, editorials, or comments

(6) Data were excluded in a duplicate publication

(7) Studies were not conducted on human subjects

2.3. Selection of Studies. The titles and abstracts of all studies derived from the search results were read thoroughly to confirm eligibility, and the full-text versions of all potentially eligible studies were then retrieved for further assessment. Doubts were discussed with a third investigator (Guolin $\mathrm{Wu}$ ). The authors of the studies were contacted for further details, if necessary.

2.4. Data Extraction. We extracted data on the authors, year of publication, country, number of patients, sensitivity, specificity, cutoff points, and area under the receiver operating characteristic (AUROC) curve from the selected studies (Table 2).

2.5. Assessment of Methodological Quality. The quality of each study was assessed according to the QUADAS (quality assessment of studies of diagnostic accuracy included in systematic reviews) checklist. Each of the 14 items in the QUADAS checklists was as "yes," "no," or "unclear" [30]. Two items were not assessed, one of which was "Were uninterpretable/intermediate results reported?" This question was not assessed because the concentration of DCP was determined by test kits; thus, there were no uninterpretable/intermediate results. The other unassessed item was that of "Withdrawals explained?" This item was not assessed because all the retrospective studies were evaluated without consideration of withdrawals. Twelve items of the QUADAS checklist are shown, and the quality of the studies are presented in Table 3.

2.6. Indices of Diagnostic Efficacy. The indices of diagnostic efficacy included sensitivity, specificity, positive likelihood ratio (PLR), negative likelihood ratio (NLR), diagnostic odds ratio (DOR), and summary receiver operating characteristic (sROC) curve.

2.7. Data Analysis. For the retrospective evaluation of our previous study, the analysis of the receiver operating characteristic (ROC) curve was applied to measure diagnostic efficacy. The cutoff points and AUROC were calculated using SPSS 17.0 software.

For the meta-analysis, a funnel plot was constructed and $P$ values were calculated. Publication bias existed when a $P$ $<0.05$ was observed. The pooled sensitivity, specificity, PLR, NLR, and DOR were summarized, and the AUROC was calculated. The statistical analyses of the meta-analysis were accomplished, using Stata 13.1 software. 
TABLE 3: Summary of methodological quality of the included studies on the basis of the review authors' judgments on the items in the QUADAS checklist for each study.

\begin{tabular}{|c|c|c|c|c|c|c|}
\hline \multirow{2}{*}{ QUADAS } & \multicolumn{6}{|c|}{ Number } \\
\hline & 1 & 2 & 3 & 4 & 5 & 6 \\
\hline Representative patient spectrum? & No & No & No & No & No & No \\
\hline Selection criteria & Unclear & Yes & Unclear & Unclear & Yes & Yes \\
\hline Acceptable reference standard? & Yes & Yes & Yes & Yes & Yes & Yes \\
\hline Acceptable delay between tests? & Yes & Yes & Yes & Yes & Yes & Yes \\
\hline Partial verification avoided? & Yes & Yes & Yes & Yes & Yes & Yes \\
\hline Differential verification avoided? & Yes & Yes & Yes & Yes & Yes & Yes \\
\hline Incorporation avoided? & Yes & Yes & Yes & Yes & Yes & Yes \\
\hline Index test execution & Yes & Yes & Yes & Yes & Yes & Yes \\
\hline Reference standard execution & Yes & Yes & Yes & Yes & Yes & Yes \\
\hline Reference standard results blinded? & Yes & Yes & Yes & Yes & Yes & Yes \\
\hline Index test results blinded? & Yes & Yes & Yes & Yes & Yes & Yes \\
\hline Relevant clinical information? & Yes & Yes & Yes & Yes & Yes & Yes \\
\hline Quality of the studies & A & A & A & A & A & A \\
\hline
\end{tabular}

\section{Results}

3.1. Characteristic of the Selected Studies. A total of 144 studies were identified from the searches conducted, of which five $[9,24,31-33]$ studies were considered suitable for inclusion in the meta-analysis, after exclusion of reviews, case reports, duplications, conference proceedings, and other unsuitable studies. All included studies were published in English. Another suitable study of ours conducted on 459 patients was included. As shown in Table 1, 2472 patients from six studies were eventually included for the meta-analysis.

3.2. Quality of the Studies. The results of the QUADAS assessment are presented in Table 2. All included studies adopted a retrospective design, and this was not regarded as the representative of the patient spectrum. In addition, three $[24,31,32]$ studies did not clearly describe the exclusion criteria applied. However, other items were effectively reported, and all six studies scored an "A."

3.3. Analysis of Publication Bias. A funnel plot was used to examine publication bias. As shown in Figure 2, the $P$ value was 0.92 , indicating the absence of publication bias among the six studies.

3.4. Heterogeneity Analysis. The analysis of heterogeneity is presented in Figure 3. The studies could have been incorporated with an index of correlation (mixed model) of 0.33 , and the proportion of heterogeneity was likely due to the threshold effect, which was 0.11 .

3.5. Meta-Analysis. The pooled sensitivity, specificity, PLR, NLR, and DOR were 0.71 (95\% CI: 0.59, 0.80), 0.93 (95\% CI: 0.87, 0.96), 9.5 (95\% CI: 5.2, 17.5), 0.32 (95\% CI: 0.22 , $0.46)$, and 30 (95\% CI: 13, 72), respectively. The AUROC curve is presented in Figure 3 with a value of 0.91 (95\% CI: $0.88,0.93)$.

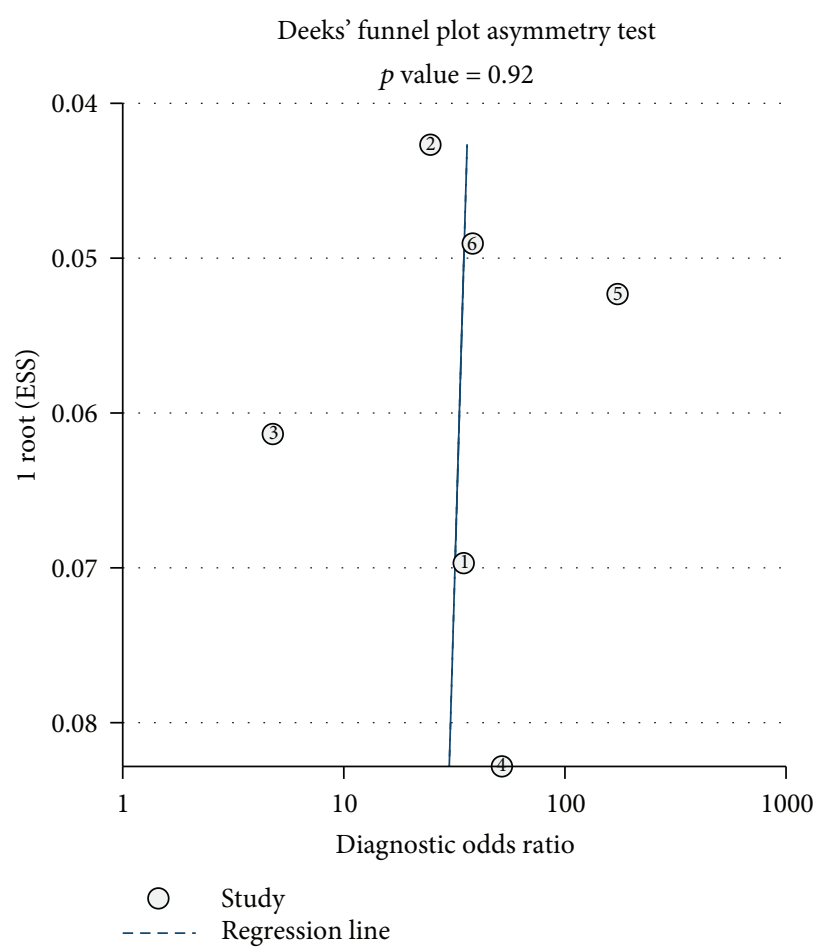

FIgURE 2: The funnel plot of publication bias. Each dot represented one study. The dots were plotted near the regression line. The funnel plot showed no publication bias among the six studies $(P=0.92)$.

\section{Discussion}

The meta-analysis of the six studies confirmed the diagnostic efficacy of DCP in patients with HBV-related HCC. All six studies were published recently (three being published in 2017). In addition, each of the studies included in the metaanalysis was of high quality and scored an "A" according to the QUADAS checklist. All serum samples were measured by enzyme immunoassay; however, limitations must still be 


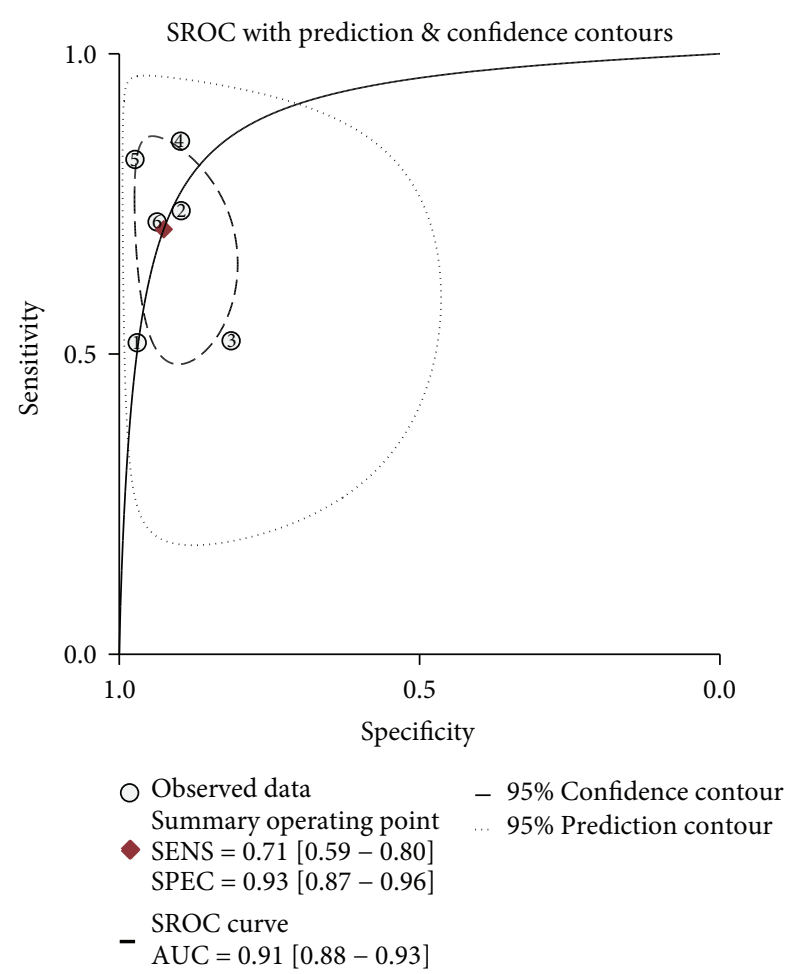

Figure 3: The sROC curve of DCP. Each dot represented one study. The red one represented the summary operating point with the high sensitivity (0.71) and specificity (0.93). The summary receiver operating characteristic (sROC) curve showed excellent diagnostic efficacy with the area under the receiver operating characteristic (AUROC) curve up to 0.91 (close to 1).

acknowledged. Firstly, all six studies were retrospective, which could increase the representative patient spectrum. Secondly, the cutoff points differed among the studies. The $40 \mathrm{mAU} / \mathrm{mL}$ cutoff point in the serum had been established in the population with heterogeneous etiologies [19]. The same cutoff point of $40 \mathrm{mAU} / \mathrm{mL}$ was employed in three studies $[9,24,31]$ (two from Korea and one from China), whereas the cutoff points of $32.09 \mathrm{mAU} / \mathrm{mL}$ [32] and $40.5 \mathrm{~m} \mathrm{AU} / \mathrm{mL}$ [33] were employed in the other two studies (both from China). The study with the lowest cutoff point of $32.09 \mathrm{mAU} / \mathrm{mL}$ [32] investigated early-stage HBV-related HCC. In our own study, the cutoff point was $49.05 \mathrm{mAU} /$ $\mathrm{mL}$. This value was the highest among the six studies. One of the main reasons for this relatively high cutoff point was the fact that all HCC cases in our study were diagnosed by histological examination. In comparison with other studies, in which diagnosis was made by histological examination or imaging characteristics, histological examination was considered the gold standard with the highest diagnostic sensitivity and specificity for all cancers' diagnosis [34]. The imaging diagnosis also takes an important role in diagnosis, but sometimes it is discordant with histological examination [35], especially in early HCC [36]. As a general rule, due to the high negative predictive value, reference values defining "below the cutoff point" biomarker concentrations (such as the 99th percentile of a healthy reference population with a coefficient of variation of $<10 \%$ ) are especially useful for diagnostic purposes [37]. With the gold standard of histological examination, the highest cutoff point of our study represents the most accurate level of DCP in HBV-related HCC.

DCP is produced by HCC and can conversely stimulate the growth and invasion of HCC through different signal pathways [38]. The diagnostic efficacy of histological examination has been confirmed for HCC. However, most of the previous studies were conducted in populations with heterogeneous liver diseases, and the respective sensitivities, specificities, and cutoff points were inconsistent among the various studies [21]. The $40 \mathrm{mAU} / \mathrm{mL}$ cutoff point has also been confirmed in populations with heterogeneous etiologies predominantly infected with HCV $[12-20,39]$. The present study, to our knowledge, is the first to report a meta-analysis that evaluates the diagnostic efficacy of DCP in the detection of HCC with homogeneous etiologies (HBV infection).

The sensitivity, specificity, PLR, NLR, DOR, and AUROC are all indices of diagnostic efficacy. The values of sensitivity, specificity, and the AUROC that are closer to 1 are preferred. A likelihood ratio greater than 1 indicates that the test result is associated with the disease. The value of a DOR ranges from 0 to infinity, and the higher values indicate better diagnostic efficacy. In the present meta-analysis, we included six studies of $2472 \mathrm{HBV}$-infected patients from China and Korea. We found that in the HBV-infected population, the pooled sensitivity, specificity, PLR, NLR, and DOR were $0.71,0.93,9.5,0.32$, and 30 , respectively. The AUROC was as high as 0.91 . The AUROC of DCP were $0.89,0.797$, and 0.893 in three meta-analyses of predominantly HCVinfected populations $[29,40,41]$. In comparison with the meta-analysis of populations with heterogeneous etiologies, the AUROC value was higher in HBV-related HCC. The diagnostic efficacy of DCP was more favorable in HBVrelated HCC.

In various guidelines for HCC worldwide, DCP has only been recommended for the surveillance of HCC. For instance, in the 2013 guidelines of Japan [8], it is recommended for the surveillance of HCC caused mainly by HCV (68\%) [42]. The present results show the superior diagnostic efficacy of DCP in HBV-related HCC. In view of these findings, we suggest that DCP should be considered for the surveillance of HCC in the established guidelines of other countries and regions, especially those with a high incidence of HBV infections, such as East Asia (except Japan) and Africa [43].

Ultrasonography has been recommended for the surveillance of HCC in almost all established guidelines worldwide [8]. However, the overall sensitivity of US is only 0.593 [10]. With a sensitivity of 0.71 and specificity of 0.93 , DCP seems to be a more favorable choice than US.

With its high diagnostic efficacy, ease of use, and reproducibility, as well as its objectivity and noninvasiveness, DCP is an ideal marker that could be considered an easily accessible complement of US for the surveillance for HBVrelated HCC. As this meta-analysis was based on six retrospective studies, more prospective studies with relatively large samples are needed in the future. 


\section{Conflicts of Interest}

The authors declare no conflicts of interest.

\section{Authors' Contributions}

Jiu Chen and Guolin $\mathrm{Wu}$ were responsible for the study design, data collection, and drafting the article. Jiu Chen and Youdi Li were responsible for the data and statistical analysis. Guolin Wu and Youdi Li were responsible for revising the article and its final approval. Jiu Chen and Guolin $\mathrm{Wu}$ contributed equally to this work.

\section{Acknowledgments}

This work was supported by Zhejiang Provincial Natural Science Foundation (grant number LQ15H280005) and the Science Program of Zhejiang Provincial Population and Family Planning Commission (grant number JSW2012A013). We would like to thank Dr. Bing Liu for the assistance with the statistical analysis.

\section{References}

[1] J. Ferlay, I. Soerjomataram, R. Dikshit et al., "Cancer incidence and mortality worldwide: sources, methods and major patterns in GLOBOCAN 2012," International Journal of Cancer, vol. 136, no. 5, pp. E359-E386, 2015.

[2] J. L. Petrick, M. Braunlin, M. Laversanne, P. C. Valery, F. Bray, and K. A. McGlynn, "International trends in liver cancer incidence, overall and by histologic subtype, 1978-2007," International Journal of Cancer, vol. 139, no. 7, pp. 15341545, 2016.

[3] N. Taura, T. Ichikawa, H. Miyaaki et al., "Frequency of elevated biomarkers in patients with cryptogenic hepatocellular carcinoma," Medical Science Monitor, vol. 19, pp. 742-750, 2013.

[4] N. Tsuchiya, Y. Sawada, I. Endo, K. Saito, Y. Uemura, and T. Nakatsura, "Biomarkers for the early diagnosis of hepatocellular carcinoma," World Journal of Gastroenterology, vol. 21, no. 37, pp. 10573-10583, 2015.

[5] A. S. Befeler and A. M. Di Bisceglie, "Hepatocellular carcinoma: diagnosis and treatment," Gastroenterology, vol. 122, no. 6, pp. 1609-1619, 2002.

[6] J. Bruix and M. Sherman, "Management of hepatocellular carcinoma: an update," Hepatology, vol. 53, no. 3, pp. 1020-1022, 2011.

[7] European Association for the Study of the Liver and European Organisation for Research and Treatment of Cancer, "EASL-EORTC clinical practice guidelines: management of hepatocellular carcinoma," Journal of Hepatology, vol. 56, no. 4, pp. 908-943, 2012.

[8] P. P. Song, J. F. Xia, Y. Inagaki et al., "Controversies regarding and perspectives on clinical utility of biomarkers in hepatocellular carcinoma," World Journal of Gastroenterology, vol. 22, no. 1, pp. 262-274, 2016.

[9] S. I. Seo, H. S. Kim, W. J. Kim et al., "Diagnostic value of PIVKA-II and alpha-fetoprotein in hepatitis B virusassociated hepatocellular carcinoma," World Journal of Gastroenterology, vol. 21, no. 13, pp. 3928-3935, 2015.
[10] R. F. Hanna, V. Z. Miloushev, A. Tang et al., "Comparative 13year meta-analysis of the sensitivity and positive predictive value of ultrasound, CT, and MRI for detecting hepatocellular carcinoma," Abdominal Radiology, vol. 41, no. 1, pp. 71-90, 2016.

[11] H. A. Liebman, B. C. Furie, M. J. Tong et al., "Des-gamma-carboxy (abnormal) prothrombin as a serum marker of primary hepatocellular carcinoma," New England Journal of Medicine, vol. 310, no. 22, pp. 1427-1431, 1984.

[12] A. Shimizu, K. Shiraki, T. Ito et al., "Sequential fluctuation pattern of serum des-gamma-carboxy prothrombin levels detected by high-sensitive electrochemiluminescence system as an early predictive marker for hepatocellular carcinoma in patients with cirrhosis," International Journal of Molecular Medicine, vol. 9, no. 3, pp. 245-250, 2002.

[13] M. L. Volk, J. C. Hernandez, G. L. Su, A. S. Lok, and J. A. Marrero, "Risk factors for hepatocellular carcinoma may impair the performance of biomarkers: a comparison of AFP, DCP, and AFP-L3," Cancer Biomarkers, vol. 3, no. 2, pp. 79-87, 2007.

[14] R. K. Sterling, L. Jeffers, F. Gordon et al., "Utility of Lens culinaris agglutinin-reactive fraction of alpha-fetoprotein and des-gamma-carboxy prothrombin, alone or in combination, as biomarkers for hepatocellular carcinoma," Clinical Gastroenterology and Hepatology, vol. 7, no. 1, pp. 104-113, 2009.

[15] J. Gao, X. Feng, Y. Inagaki et al., "Des- $\gamma$-carboxy prothrombin and c-Met were concurrently and extensively expressed in hepatocellular carcinoma and associated with tumor recurrence," BioScience Trends, vol. 6, no. 4, pp. 153-159, 2012.

[16] H. Okuda, T. Nakanishi, K. Takatsu et al., "Serum levels of desgamma-carboxy prothrombin measured using the revised enzyme immunoassay kit with increased sensitivity in relation to clinicopathologic features of solitary hepatocellular carcinoma," Cancer, vol. 88, no. 3, pp. 544-549, 2000.

[17] J. A. Marrero, G. L. Su, W. Wei et al., "Des-gamma carboxyprothrombin can differentiate hepatocellular carcinoma from nonmalignant chronic liver disease in American patients," Hepatology, vol. 37, no. 5, pp. 1114-1121, 2003.

[18] J. A. Marrero, Z. Feng, Y. Wang et al., "Alpha-fetoprotein, desgamma carboxyprothrombin, and lectin-bound alphafetoprotein in early hepatocellular carcinoma," Gastroenterology, vol. 137, no. 1, pp. 110-118, 2009.

[19] C. Li, Z. Zhang, P. Zhang, and J. Liu, "Diagnostic accuracy of des-gamma-carboxy prothrombin versus a-fetoprotein for hepatocellular carcinoma: a systematic review," Hepatology Research, vol. 44, no. 10, pp. E11-E25, 2014.

[20] R. Tateishi, H. Yoshida, Y. Matsuyama, N. Mine, Y. Kondo, and M. Omata, "Diagnostic accuracy of tumor markers for hepatocellular carcinoma: a systematic review," Hepatology International, vol. 2, no. 1, pp. 17-30, 2008.

[21] G. J. Li, Q. Y. Chen, T. J. Harrison et al., "Des- $\gamma$ carboxyprothrombin may not be a good biomarker for hepatocellular carcinoma in those chronically infected with hepatitis B virus with basal core promoter double mutations $\left(T^{\wedge}\{1762\}\right.$, $\mathrm{A} \wedge\{1764\})$, a prospective study," Cancer Biomarkers, vol. 18, no. 3, pp. 241-248, 2017.

[22] European Association for the Study of the Liver and European Organisation for Research and Treatment of Cancer, "EASL-EORTC clinical practice guidelines: management of hepatocellular carcinoma," European Journal of Cancer, vol. 48, no. 5, pp. 599-641, 2012. 
[23] J. D. Stanaway, A. D. Flaxman, M. Naghavi et al., "The global burden of viral hepatitis from 1990 to 2013: findings from the Global Burden of Disease Study 2013," Lancet, vol. 388, no. 10049, pp. 1081-1088, 2016.

[24] S. Huang, F. Jiang, Y. Wang et al., "Diagnostic performance of tumor markers AFP and PIVKA-II in Chinese hepatocellular carcinoma patients," Tumor Biology, vol. 39, no. 6, 2017.

[25] H. S. Kim, J. W. Park, J. S. Jang et al., "Prognostic values of alpha-fetoprotein and protein induced by vitamin $\mathrm{K}$ absence or antagonist-II in hepatitis B virus-related hepatocellular carcinoma: a prospective study," Journal of Clinical Gastroenterology, vol. 43, no. 5, pp. 482-488, 2009.

[26] X. Feng, P. Song, P. Bie et al., "Des- $\gamma$-carboxyprothrombin plasma level in diagnosis of hepatocellular carcinoma in a Chinese population undergoing surgery," Medical Science Monitor, vol. 22, pp. 1663-1672, 2016.

[27] J. Ji, H. Wang, Y. Li et al., "Diagnostic evaluation of desgamma-carboxy prothrombin versus $\alpha$-fetoprotein for hepatitis B virus-related hepatocellular carcinoma in China: a large-scale, multicentre study," PLoS One, vol. 11, no. 4, article e0153227, 2016.

[28] M. Meguro, T. Mizuguchi, T. Nishidate et al., "Prognostic roles of preoperative $\alpha$-fetoprotein and des- $\gamma$-carboxy prothrombin in hepatocellular carcinoma patients," World Journal of Gastroenterology, vol. 21, no. 16, pp. 4933-4945, 2015.

[29] P. Gao, M. Li, Q. B. Tian, and D. W. Liu, "Diagnostic performance of des- $\gamma$-carboxy prothrombin (DCP) for hepatocellular carcinoma: a bivariate meta-analysis," Neoplasma, vol. 59, no. 2, pp. 150-159, 2012.

[30] P. Whiting, A. W. Rutjes, J. B. Reitsma, P. M. M. Bossuyt, and J. Kleijnen, "The development of QUADAS: a tool for the quality assessment of studies of diagnostic accuracy included in systematic reviews," BMC Medical Research Methodology, vol. 3, no. 1, p. 25, 2003.

[31] Y. J. Yoon, K. H. Han, and D. Y. Kim, "Role of serum prothrombin induced by vitamin $\mathrm{K}$ absence or antagonist-II in the early detection of hepatocellular carcinoma in patients with chronic hepatitis B virus infection," Scandinavian Journal of Gastroenterology, vol. 44, no. 7, pp. 861-866, 2009.

[32] X. Wang, W. Zhang, Y. Liu et al., "Diagnostic value of prothrombin induced by the absence of vitamin $\mathrm{K}$ or antagonist-II (PIVKA-II) for early stage HBV related hepatocellular carcinoma," Infectious Agents and Cancer, vol. 12, no. 1, p. 47, 2017.

[33] X. Q. Tang, H. Li, L. B. Yan et al., "Diagnostic value of PIVKAII in detecting hepatocellular carcinoma," Future Virology, vol. 12, no. 5, pp. 259-267, 2017.

[34] F. Pezzuto, L. Buonaguro, F. Buonaguro, and M. Tornesello, "The role of circulating free DNA and microRNA in noninvasive diagnosis of HBV- and HCV-related hepatocellular carcinoma," International Journal of Molecular Sciences, vol. 19 , no. 4, 2018.

[35] L. Liberman, M. Drotman, E. A. Morris et al., "Imaging-histologic discordance at percutaneous breast biopsy," Cancer, vol. 89, no. 12, pp. 2538-2546, 2000.

[36] J. Y. Choi, J. M. Lee, and C. B. Sirlin, "CT and MR imaging diagnosis and staging of hepatocellular carcinoma: part I. Development, growth, and spread: key pathologic and imaging aspects," Radiology, vol. 272, no. 3, pp. 635-654, 2014.

[37] A. Kaeberich, V. Seeber, D. Jiménez et al., “Age-adjusted highsensitivity troponin $\mathrm{T}$ cut-off value for risk stratification of pulmonary embolism," European Respiratory Journal, vol. 45, no. 5, pp. 1323-1331, 2015.

[38] S. X. Cui, X. F. Yu, and X. J. Qu, "Roles and signaling pathways of des- $\gamma$-carboxyprothrombin in the progression of hepatocellular carcinoma," Cancer Investigation, vol. 34, no. 9, pp. 459464, 2016

[39] F. A. Durazo, L. M. Blatt, W. G. Corey et al., "Des-gamma-carboxyprothrombin, alpha-fetoprotein and AFP-L3 in patients with chronic hepatitis, cirrhosis and hepatocellular carcinoma," Journal of Gastroenterology and Hepatology, vol. 23, no. 10, pp. 1541-1548, 2008.

[40] B. Hu, X. Tian, J. Sun, and X. Meng, "Evaluation of individual and combined applications of serum biomarkers for diagnosis of hepatocellular carcinoma: a meta-analysis," International Journal of Molecular Sciences, vol. 14, no. 12, pp. 2355923580, 2013.

[41] R. Zhu, J. Yang, L. Xu et al., "Diagnostic performance of des- $\gamma$-carboxy prothrombin for hepatocellular carcinoma: a meta-analysis," Gastroenterology Research and Practice, vol. 2014, Article ID 529314, 9 pages, 2014.

[42] S. Ashtari, M. A. Pourhoseingholi, A. Sharifian, and M. R. Zali, "Hepatocellular carcinoma in Asia: prevention strategy and planning," World Journal of Hepatology, vol. 7, no. 12, pp. 1708-1717, 2015.

[43] A. Ozakyol, "Global epidemiology of hepatocellular carcinoma (HCC epidemiology)," Journal of Gastrointestinal Cancer, vol. 48, no. 3, pp. 238-240, 2017. 


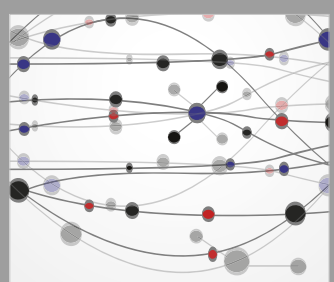

The Scientific World Journal
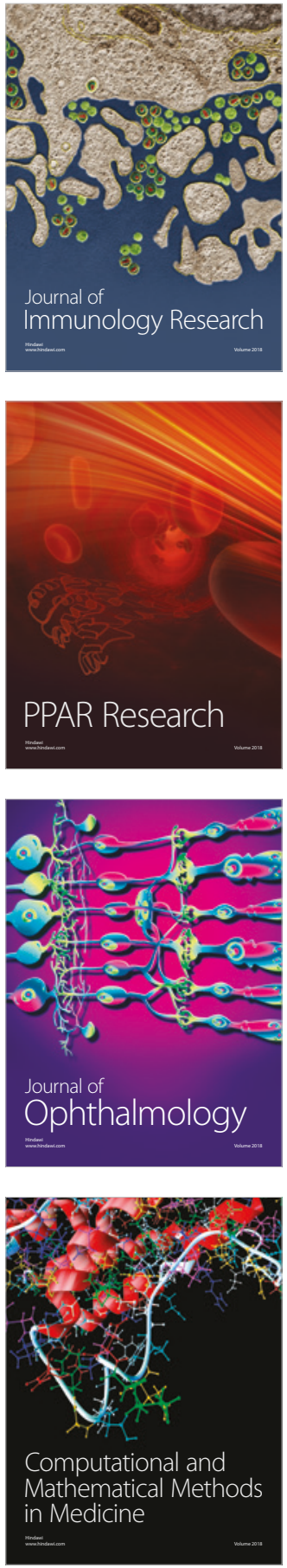

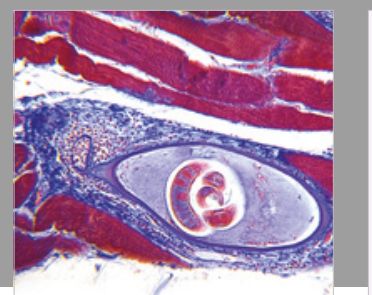

Gastroenterology Research and Practice

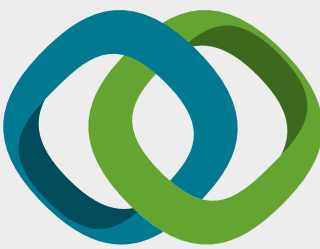

\section{Hindawi}

Submit your manuscripts at

www.hindawi.com
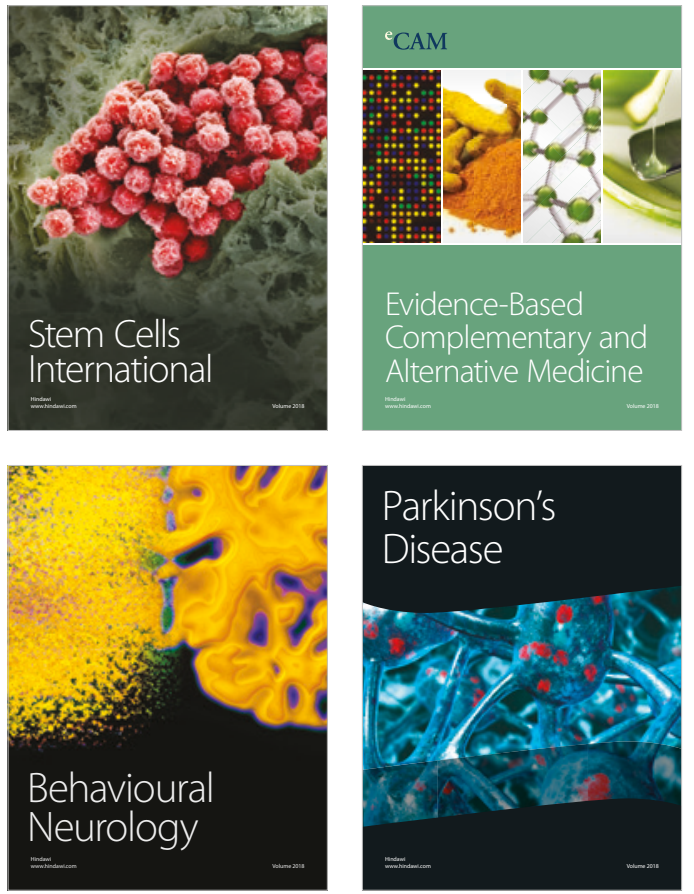

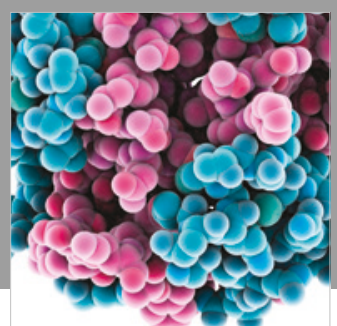

ournal of

Diabetes Research

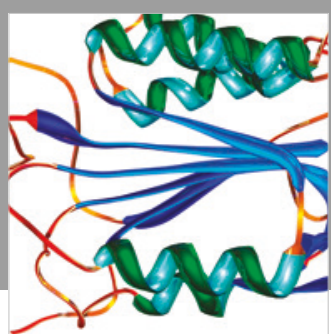

Disease Markers
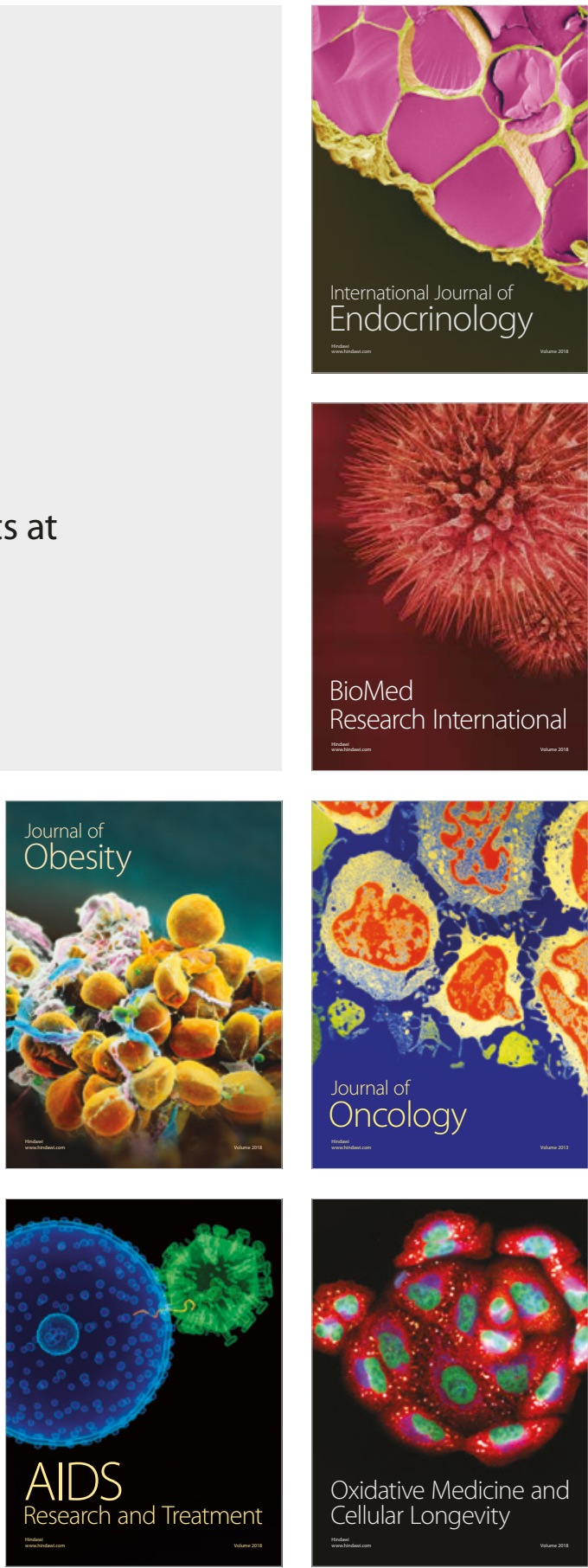